\section{WIRTSCHAFTS INFORMATIK}

Gegenstand der Zeitschrift sind Forschungsergebnisse im Bereich der Wirtschaftsinformatik und Praxisbeispiele von fortschrittlichen Anwendungen. Konkrete Lösungen für technologiegestützte Anwendungssysteme werden nur dann publiziert, wenn sie Modellcharakter auch für andere Anwendungen besitzen.

Wichtige Randgebiete werden ebenfalls abgedeckt, soweit die Entwicklungen im Bereich der engeren Wirtschaftsinformatik wesentlich betroffen sind Beispiele hierfür sind; die Wirkung der Informatik auf Wirtschaft, Individuum und Gesellschaft sowie Fragen der Ausund Weiterbildung.

Die Zeitschrift wendet sich an Leser in Wissenschaft und Praxis, die auf dem Gebiet der Wirtschaftsinformatik arbeiten. Besonders sind auch Studierende und andere Personen angesprochen, die den Anschluss an die modernen Entwicklungen in diesem Fach suchen.

In der Zeitschrift werden wissenschaftliche Originalbeiträge publiziert. Die Aufsätze werden einem anonymen Begutachtungsverfahren unterworfen. Ein Teil der Hefte wird einem Schwerpunktthema gewidmet.

Es sind derzeit folgende Rubriken eingerichtet (in Klammern die verantwortlichen Herausgeber):

\section{Rubriken}

WI - State-of-the-Art (Chamoni)

WI - Innovative Produkte (Winand')

WI - Für Sie gesurft (Becker)

WI - Schlagwort (Sinz)

WI - Interview (Heinzl)

WI - Für Sie gelesen (Müller)

WI - Vergleichende Buchbesprechung (Frank)

WI - Buchbesprechungen (Graver ${ }^{2}$ )

WI - Dissertationen (Buxmann)

WI - Studierendenforum (Weinhardt)

Meinung/Dialog (Buhl)

Mitteilungen des GI-FB WI (Oberweis)

Mitteilungen der WKWI (Heinzl)

Aus den Hochschulen (Hofmann/König/

Rump)

WI - Online (Loos)

1 Prof. Dr. U. Winand, Universität Kassel

2 Prof. Dr. M. Graver, Universität Siegen

\section{Geschäftsführender Herausgeber}

Prof. Dr. Wolfgang König, Institut für Wirtschaftsinformatik,

Universität Frankfurt, Mertonstraße 17, 60054 Frankfurt (Main)

Telefon 069 798-28594, Telefax 069 798-28585

E-Mail: koenig@wiwi.uni-frankfurt.de

\section{Herausgeber}

Prof. Dr. H.-J. Appelrath, Universität Oldenburg

R. Bauer, IBM Deutschland GmbH, Stuttgart

Prof. Dr. J. Becker, Universität Münster

Prof. Dr. H. U. Buhl, Universität Augsburg

Prof. Dr. P. Buxmann, TU Darmstadt

Prof. Dr. P. Chamoni, Universität Duisburg-Essen

K.-H. Flöther, Accenture, Kronberg

Prof. Dr. U. Frank, Universität Duisburg-Essen

Prof. Dr. U. Hasenkamp, Universität Marburg

Prof. Dr. A. Heinzl, Universität Mannheim

Prof. Dr. G. R. Hofmann, FH Aschaffenburg

Prof. Dr. D. Karagiannis, Universität Wien

Prof. Dr. S. Kirn, Universität Hohenheim

Prof. Dr. M. Kleinaltenkamp, FU Berlin

Dr. J. Laartz, McKinsey \& Company, Düsseldorf

H.-J. Lamberti, Deutsche Bank AG, Frankfurt (Main)

Prof. Dr. W. Lamersdorf, Universität Hamburg

Prof. Dr. P. Loos, Universität des Saarlandes

Prof. Dr. Dr. h. c. H. C. Mayr, Universität Klagenfurt

Prof. Dr. G. Müller, Universität Freiburg

Prof. Dr. A. Oberweis, Universität Karlsruhe

Prof. Dr. Dres. h. c. A. Picot, Universität München

Prof. Dr. F. J. Rump, FH Oldenburg/Ostfriesland/Wilhelmshaven

P. Schumann, Schenker AG, Essen

Prof. Dr. E. Sinz, Universität Bamberg

Prof. Dr. C. Weinhardt, Universität Karlsruhe

Prof. Dr. R. Winter, Universität St. Gallen

S. Wolf, IS Teledata AG, Frankfurt (Main)

Dr. P. Zencke, SAPAG, Walldorf

\section{Assoziierte Herausgeber}

Prof. Dr. W. Abramowicz, Wirtschaftsuniversität Poznan, Polen

Prof. Dr. H. Sol, Universität Groningen, Niederlande

\section{Redaktion}

Prof. Dr. Ulrich Hasenkamp, Dipl.-Kfm. Claus Häberle, Dipl.-Kff. Elizaveta Kozlova,

Dipl.-Kfm. Jens Lehmbach

Wirtschaftsinformatik, Universität Marburg

35032 Marburg

Telefon 06421 28-23894, Telefax 06421 28-26554

E-Mail: redaktion@wirtschaftsinformatik.de

Dipl.-Kfm. Rainer B. Fladung, Universität Frankfurt (Main)

E-Mail: rfladung@wiwi.uni-frankfurt.de

http://www.wirtschaftsinformatik.de 